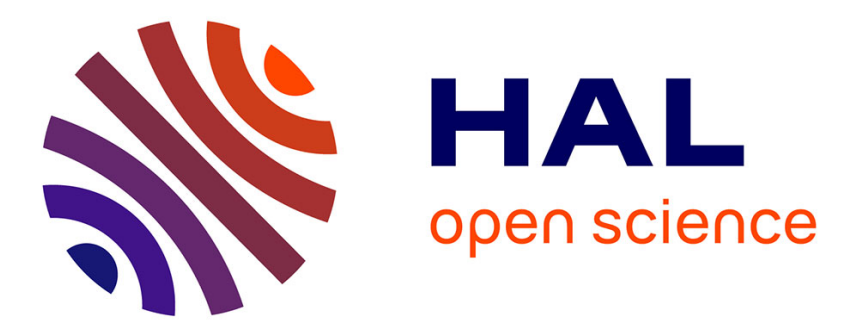

\title{
Investigation of gain ripple in two-pump fiber optical parametric amplifiers
}

Armand Vedadi, Michel E. Marhic, Eric Lantz, Hervé Maillotte, Thibaut

Sylvestre

\section{- To cite this version:}

Armand Vedadi, Michel E. Marhic, Eric Lantz, Hervé Maillotte, Thibaut Sylvestre. Investigation of gain ripple in two-pump fiber optical parametric amplifiers. Optics Letters, 2008, 33 (19), pp.22032205. hal-00362432

\section{HAL Id: hal-00362432 \\ https://hal.science/hal-00362432}

Submitted on 28 Aug 2013

HAL is a multi-disciplinary open access archive for the deposit and dissemination of scientific research documents, whether they are published or not. The documents may come from teaching and research institutions in France or abroad, or from public or private research centers.
L'archive ouverte pluridisciplinaire HAL, est destinée au dépôt et à la diffusion de documents scientifiques de niveau recherche, publiés ou non, émanant des établissements d'enseignement et de recherche français ou étrangers, des laboratoires publics ou privés. 


\title{
Investigation of gain ripple in two-pump fiber optical parametric amplifiers
}

\author{
Armand Vedadi, ${ }^{1, *}$ Michel E. Marhic, ${ }^{1}$ Eric Lantz, ${ }^{2}$ Hervé Maillotte, ${ }^{2}$ and Thibaut Sylvestre ${ }^{2}$ \\ ${ }^{1}$ Institute of Advanced Telecommunications, Swansea University, Singleton Park, Swansea SA2 8PP, Wales, UK \\ ${ }^{2}$ Département d'Optique P.M. Duffieux, Institut FEMTO-ST, Centre National de la Recherche Scientifique \\ UMR 6174, Université de Franche-Comté, 25030 Besançon, France \\ *Corresponding author: a.vedadi@swansea.ac.uk
}

Received May 21, 2008; accepted August 6, 2008;

posted August 25, 2008 (Doc. ID 96530); published September 25, 2008

By using the four-sideband theory, we analyze the gain spectrum in wideband two-pump fiber optical parametric amplifiers and predict gain ripples over the flat gain region. We derive an approximation of their pseudo-periods and discuss methods for reducing their amplitudes. (C) 2008 Optical Society of America OCIS codes: $060.0060,060.4370$.

Fiber-optical parametric amplifiers (FOPAs) are being developed for efficient ultrahigh bandwidth and ultrafast signal processing technologies such as wavelength conversion, multiplexing, sampling, buffering, etc. [1,2]. Thanks to both the ultrafast Kerr nonlinearity and the flexibility in the design of the fiber's dispersion curve, large and broadband parametric gain can be achieved in the $1.55 \mu \mathrm{m}$ region by using one or two pumps $[1,2]$. The latter architecture is of interest for telecommunication applications: It allows cancellation of signal distortion and idler broadening, improvement of polarization insensitivity, and the design of wideband amplifiers with flat gain spectra [3-7]. Indeed, using a standard four-wave mixing theory involving the two pumps, the signal, and the generated idler waves, it is possible to optimize the position of the two pumps so as to achieve wideband flat gain between the two pumps [6,7]. However, this model is not valid close to the pumps, as one has to take into account the two other sidebands that are generated in the region outside the two pumps by
Bragg scattering (BS) and modulation instability (MI). The interaction among all four sidebands leads to a gain reduction in the vicinity of the two pumps [8-10]. Recently, we showed that by taking into account the four sidebands, one can predict small ripples on the flat gain region that can lead to impairments for telecommunication applications [11]. In this Letter, we review these preliminary results linked to a recent experimental demonstration of a wideband and flat-gain two-pump FOPA (2P-FOPA) [7]. We explain in detail the mechanism of the observed ripples and discuss guidelines to minimize those ripples when designing flat-gain 2P-FOPAs.

Following notations similar to [12], we denote by $\omega_{1}, \omega_{2}$ the angular frequencies of the two pumps and by $\omega_{3}, \omega_{4}, \omega_{5}$, and $\omega_{6}$ those of signal, idler, and the sidebands generated outside the two pumps (see Fig. 1). We refer to this model as the four-sideband (4S) model. Assuming that the pumps are nondepleted and following the steps in [5], it leads to a set of four coupled equations:

$$
\frac{\partial}{\partial z}\left(\begin{array}{c}
A_{5} \\
A_{3}^{*} \\
A_{4} \\
A_{6}^{*}
\end{array}\right)=j\left(\begin{array}{ccccc}
\Delta \beta_{5}+\gamma P_{1} & \gamma P_{1} & r & r \\
-\gamma P_{1} & -\Delta \beta_{3}-\gamma P_{1} & -r & -r \\
r & \vdots \ldots \ldots \ldots \ldots \ldots & r & \Delta \beta_{4}+\gamma P_{2} & \gamma P_{2} \\
-r & -r & -\gamma P_{2} & -\Delta \beta_{6}-\gamma P_{2}
\end{array}\right)\left(\begin{array}{c}
A_{5} \\
A_{3}^{*} \\
A_{4} \\
A_{6}^{*}
\end{array}\right)
$$

This equation has the form $\partial Y / \partial z=j M Y . P_{1}, P_{2}$ are the pump powers, and $A_{3}, A_{4}, A_{5}$, and $A_{6}$ are the sidebands' slowly varying amplitudes (SVAs). $\gamma$ is the fiber nonlinearity coefficient and $r=2 \gamma \sqrt{P_{1} P_{2}}$. The terms $\Delta \beta_{i}(i=3,4,5,6)$ in $M$ represent linear wavevector mismatches between sideband $i$ and its closest pump. They can be expanded in Taylor series about $\omega_{C}=\left(\omega_{1}+\omega_{2}\right) / 2$ :

$$
\Delta \beta_{i}=\beta\left(\omega_{i}\right)-\beta\left(\omega_{g}\right)=\sum_{k=2}^{\infty}(-1)^{k \times i} \frac{\beta_{k}}{k !}\left(\Delta \omega_{i}^{k}-\Delta \omega_{P}^{k}\right),
$$

where $\quad \Delta \omega_{P}=\left(\omega_{2}-\omega_{1}\right) / 2, \quad g=\left((-1)^{\prime}+3\right) / 2, \Delta \omega_{i}=\Delta \omega_{S}$ $=\left|\omega_{3,4}-\omega_{C}\right|$ for $i=(3,4)$, and $\Delta \omega_{i}=\Delta \omega_{S B}=\omega_{C}-\omega_{5}$ $=2 \Delta \omega_{P}-\Delta \omega_{S}$ for $i=(5,6) . \beta_{k}$ are the higher-order dispersion coefficients at $\omega_{C}$. In practice, it is sufficient to consider $\beta_{2}, \beta_{3}$, and $\beta_{4}$ to account for most dispersion-related behaviors.

$M$ does not depend on $z$; hence the solution can be readily derived as follows:

$$
Y(z)=\exp (j M z) Y(0) .
$$

By setting the appropriate sideband amplitude to 1 and the others to 0 as initial conditions, the gain can 


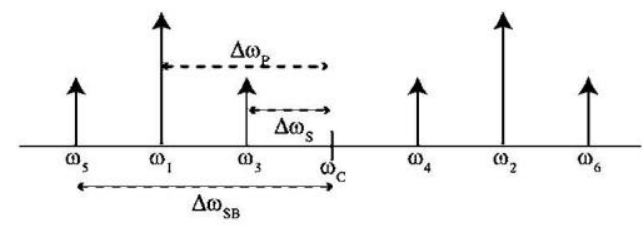

Fig. 1. Four-sideband model with pumps, signal, and three generated idlers.

be numerically calculated for signals located in $] \omega_{1}$ $-\Delta \omega_{P}, \omega_{2}+\Delta \omega_{P}[$.

To obtain a closed-form analytical solution for the parametric gain, one would need to calculate the eigenvalues and eigenvectors of $M$. This has been done for signals located in the vicinity of one pump [12], but the calculation remains complex for the whole spectrum. It is therefore convenient to neglect sidebands $A_{5}$ and $A_{6}$. Using this two-sideband (2S) model, $M$ is reduced to the $2 \times 2$ matrix framed in dotted line in Eq. (1). The signal output SVA can then be derived as in Section A1.2 of [1], with the following result:

$$
\begin{aligned}
A_{i}= & \exp \left\{(-1)^{i} j\left[\gamma\left(P_{2}-P_{1}\right) / 2+\left(\beta_{3} / 6\right)\left(\Delta \omega_{S}^{3}-\Delta \omega_{P}^{3}\right)\right] z\right\} \\
& \times\left[\cosh (g z)-j \frac{\kappa}{2 g} \sinh (g z)\right] \quad(i=3,4),
\end{aligned}
$$

where $\kappa=\gamma\left(P_{1}+P_{2}\right)+2 \Sigma_{k=1}^{\infty} \beta_{2 k} /(2 k) !\left(\Delta \omega_{S} 2 k-\Delta \omega_{P}^{2 k}\right)$ is the total wave-vector mismatch between the four interacting waves, $g=\sqrt{r^{2}-(\kappa / 2)^{2}}$, and $r=2 \gamma \sqrt{P_{1} P_{2}}$. For high gain, one can neglect the decaying exponential in Eq. (4):

$$
\begin{aligned}
A_{i} \approx & \left(\frac{1}{2}-j \frac{\kappa}{4 g}\right) \times \exp \left(\left\{( - 1 ) ^ { i } j \left[\gamma\left(P_{2}-P_{1}\right) / 2\right.\right.\right. \\
& \left.\left.\left.+\left(\beta_{3} / 6\right)\left(\Delta \omega_{S}^{3}-\Delta \omega_{P}^{3}\right)\right]+g\right\} z\right) \quad(i=3,4) .
\end{aligned}
$$

The signal gain is also straightforwardly derived from Eq. (4) as $G=1+[(r / g) \sinh (g L)]^{2}$.

Using the $2 \mathrm{~S}$ and $4 \mathrm{~S}$ models, we analyzed an experimental 2P-FOPA with flat gain spectrum over more than $70 \mathrm{~nm}$ demonstrated by Chavez Boggio et al. in a highly nonlinear fiber of $243 \mathrm{~m}$ effective length [7]. The results are shown in Fig. 2. The $2 \mathrm{~S}$ model, which was used to design a flat-gain FOPA, shows good agreement with the experimental results for signals that are separated by more than $10 \mathrm{~nm}$ from the closest pump. In particular, the flat-gain re-

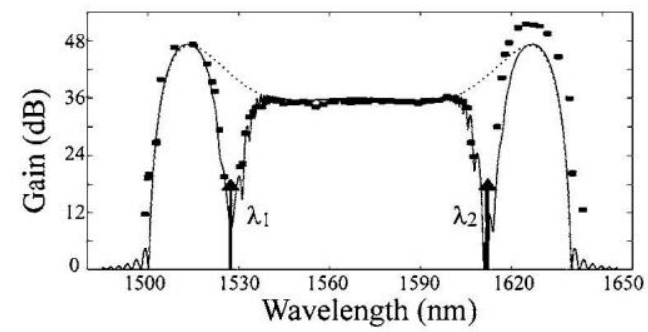

Fig. 2. Spectrum of the 2P-FOPA gain. Squares, experimental results; solid curve, $4 \mathrm{~S}$ analysis; dashed curve, $2 \mathrm{~S}$ analysis (standard model). The parameters are $\beta_{2}$ $=6.4 \times 10^{-30} \mathrm{~s}^{2} \mathrm{~m}^{-1}, \quad \beta_{3}=0.65 \times 10^{-40} \mathrm{~s}^{3} \mathrm{~m}^{-1}, \quad \beta_{4}=-1.6$ $\times 10^{-55} \mathrm{~s}^{4} \mathrm{~m}^{-1}, \gamma=8 \mathrm{~W}^{-1} \mathrm{~m}^{-1}$, and $\Delta \omega_{P}=3.25 \times 10^{13} \mathrm{rad} / \mathrm{s}$. gion of $70 \mathrm{~nm}$ is fairly well fitted. As expected, the reduction of the parametric gain in the vicinity of the pumps can be fitted by using the $4 \mathrm{~S}$ model. The discrepancy observed for wavelengths larger than $\lambda_{2}$ is due to Raman gain. However, when zooming in on the edges of the flat region of the spectrum, Fig. 3 shows that the $4 \mathrm{~S}$ model predicts gain ripples. Clearly, these ripples occur in a region where both MI and BS processes are negligible. Their existence was verified by simulation of the nonlinear Schrödinger equation. The pseudo-periods of the ripples range from 0.5 to $1 \mathrm{~nm}$, and their amplitudes can be larger than $1.5 \mathrm{~dB}$, which is sufficient to impair telecommunication applications [11].

Equation (3) gives very little insight about the underlying mechanisms of these ripples, as it can be solved only by numerical means. Instead, we will limit our discussion to qualitative considerations within the flat-gain region (where the approximation of the two sidebands model is good). The four complex eigenvalues of $M$ have the form $\lambda_{3,4}\left(\Delta \omega_{S}\right)$ $=p_{1}\left(\Delta \omega_{S}\right) \pm j g_{1}\left(\Delta \omega_{S}\right) \quad$ and $\quad \lambda_{5,6}\left(\Delta \omega_{S}\right)=p_{2}\left(\Delta \omega_{S}\right)$ $\pm j g_{2}\left(\Delta \omega_{S}\right)$. The output field amplitude of the signal is a linear combination of four exponentials $\exp \left(j \lambda_{i} z\right)(i$ $=3,4,5,6$ ), with weights that depend on the initial field amplitudes. Neglecting all decaying exponentials, the output SVA is approximated by

$$
A \approx a \exp \left(j p_{1} z+g_{1} z\right)+b \exp \left(j p_{2} z+g_{2} z\right),
$$

and thus the parametric gain by

$$
\begin{aligned}
G(z) \approx & |a|^{2} e^{2 g_{1} z}+|b|^{2} e^{2 g_{2} z}+2|a b| e^{\left(g_{1}+g_{2}\right) z} \\
& \times \cos \left[\left(p_{1}-p_{2}\right) z+\varphi\right],
\end{aligned}
$$

where $\varphi=\arg \left(a b^{*}\right)$ and $a, b$ are weights. This latter expression shows how the different eigenvalues combine to give the output parametric gain. It also shows that $G$ contains an oscillating term whose pseudoperiod depends on $z$. In the region of flat gain that is of interest for telecommunications, Eq. (5) also shows that the output field is approximated by a single exponential. Because the exponentials are a basis for function decomposition, we infer that one of the terms [say $a \exp \left(j p_{1} z+g_{1} z\right)$ ] in Eq. (6) should be dominant and equal to $A_{i}$ of Eq. (5), while the other term is a small perturbation $(|a| \gg|b|)$. In Eq. (7), the second term could therefore be neglected, and the third term would account for the small ripples that are observed. By assumption, we also get $p_{1}\left(\Delta \omega_{S}\right)$ $=(-1)^{i}\left[\gamma\left(P_{2}-P_{1}\right) / 2+\left(\beta_{3} / 6\right)\left(\Delta \omega_{S}^{3}-\Delta \omega_{P}^{3}\right)\right] z(i=3,4)$ and $g_{1}=g$. We note that $\Delta \beta_{3,4}\left(\Delta \omega_{S}\right)=\Delta \beta_{5,6}\left(\Delta \omega_{S B}\right)$. Suppose that $\lambda_{3}\left(\Delta \omega_{S}\right)$ is an eigenvalue of $M$ with the associ-

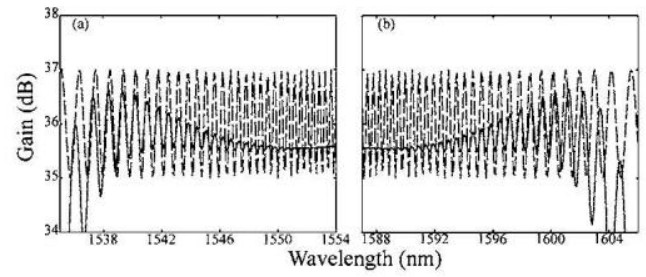

Fig. 3. (a), (b) Zoom in on the edges of the flat-gain region. Dashed curve, plot of Eq. (9). 
ated eigenvector ${ }^{T}\left[v_{5}\left(\Delta \omega_{S}\right) v_{3}\left(\Delta \omega_{S}\right) v_{4}\left(\Delta \omega_{S}\right) v_{6}\left(\Delta \omega_{S}\right)\right]$. From the second row of $M$ it then comes that

$$
\begin{aligned}
\left(\Delta \beta_{3}\left(\Delta \omega_{S B}\right)+\gamma P_{1}\right) \times v_{3}\left(\Delta \omega_{S B}\right)+\gamma P_{1} \times v_{5}\left(\Delta \omega_{S B}\right) \\
\quad+r \times v_{6}\left(\Delta \omega_{S B}\right)+r \times v_{4}\left(\Delta \omega_{S B}\right) \\
=\left(\Delta \beta_{5}\left(\Delta \omega_{S}\right)+\gamma P_{1}\right) \times v_{3}\left(\Delta \omega_{S B}\right)+\gamma P_{1} \times v_{5}\left(\Delta \omega_{S B}\right) \\
\quad+r \times v_{6}\left(\Delta \omega_{S B}\right)+r \times v_{4}\left(\Delta \omega_{S B}\right) .
\end{aligned}
$$

Similar expressions hold with all other lines of $M$. Thus, we deduce that $-\lambda_{3}^{*}\left(\Delta \omega_{S B}\right)$ is another eigenvalue of $M$ with associated eigenvector ${ }^{T}\left[v_{3}\left(\Delta \omega_{S B}\right) v_{5}\left(\Delta \omega_{S B}\right) v_{6}\left(\Delta \omega_{S B}\right) v_{4}\left(\Delta \omega_{S B}\right)\right]$. Identifying the real and imaginary parts with $\lambda_{5}\left(\Delta \omega_{S}\right)$, we deduce $p_{2}\left(\Delta \omega_{S}\right)=-p_{1}\left(\Delta \omega_{S B}\right)$ and $g_{2}\left(\Delta \omega_{S}\right)=g_{1}\left(\Delta \omega_{S B}\right)$. Hence

$$
\begin{aligned}
\cos \left[\left(p_{1}-p_{2}\right) z\right] \approx & \cos \left\lfloor\beta_{3} \Delta \omega_{P}\left(\Delta \omega_{P}-\Delta \omega_{S}\right)^{2} z\right. \\
& \left.+\gamma\left(P_{2}-P_{1}\right) z\right\rfloor .
\end{aligned}
$$

We have plotted $\cos \left[\beta_{3} \Delta \omega_{P}\left(\Delta \omega_{P}-\Delta \omega_{S}\right)^{2} z\right]$ with an adjusted phase in Fig. 3 as a dashed curve. It shows a fairly good agreement with the pseudo-periods of the ripples within the flat region. Equation (9) also shows the influence of the fiber dispersion slope and length on the ripples pseudo-period. In this region where MI and BS processes are negligible, the relative amplitude of the ripples depends on the ratio $2|b / a| \exp \left\{\left[g\left(\Delta \omega_{S B}\right)-g\left(\Delta \omega_{S}\right)\right] z\right\}$. Although $b / a$ is very small, this latter expression shows that noticeable ripples may occur if $g$ in the inner band between the two pumps (at $\pm \Delta \omega_{S}$ ) is smaller than or equal to $g$ for a signal located at the symmetrical frequency relatively to the closest pump [at $\pm \Delta \omega_{S B}= \pm\left(2 \Delta \omega_{P}-\Delta \omega_{S}\right)$ ].

The maxima of $g$ correspond to the roots of $\kappa=0$. When $\beta_{4}>0$, one can choose $\beta_{2}$ and $\Delta \omega_{P}$ such that $\kappa$ can be written in terms of a fourth-order Chebyshev polynomial [6] whose roots are all located between the two pumps. As a consequence, $g$ is always greater in the inner band between the two pumps than in the outer band and the ripples become negligible. When $\beta_{4}<0$, two roots $\pm \Omega_{1}$ of $\kappa=0$ are always located in the outer band [7]. To minimize the ripples, one should therefore design the FOPA spectrum such that $\kappa$ possesses two other roots located close to $\pm 2 \Delta \omega_{P}-\Omega_{1}$. To verify these assumptions, we have plotted in Fig. 4(a) the spectra of two different 2P-FOPAs: one with positive $\beta_{4}$ and the other one with a negative $\beta_{4}$ and an optimized $\beta_{2}$. All other parameters are the same as for the FOPA studied in Figs. 2 and 3. In both cases, the parametric gain is increased in the flat region and the ripples are reduced compared with the FOPA studied in Figs. 2 and 3. To quantify the reduction of the ripples amplitudes, we have numerically calculated $|a|^{2} e^{2 g_{1} L}$ and subtracted that amount from $G(L)$. The results are shown on Fig. 4(b) and show that the relative ripples amplitudes are significantly reduced for the FOPAs of Fig. 4(a) compared with the FOPA of Figs. 2 and 3.

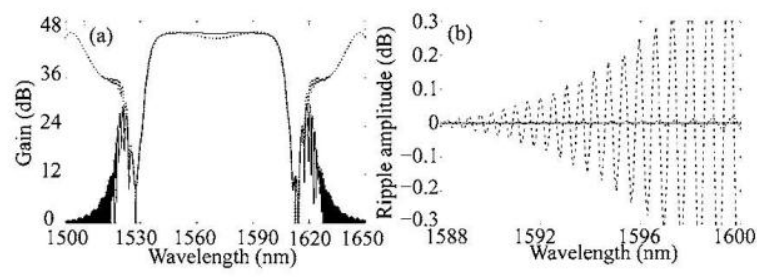

Fig. 4. (a) Gain spectra of 2P-FOPAs with $\beta_{2}=6.3$ $\times 10^{-30} \mathrm{~s}^{2} \mathrm{~m}^{-1}, \beta_{4}=1.6 \times 10^{-55} \mathrm{~s}^{4} \mathrm{~m}^{-1}$ (solid curve) and $\beta_{2}$ $=5 \times 10^{-29} \mathrm{~s}^{2} \mathrm{~m}^{-1}, \quad \beta_{4}=-1.6 \times 10^{-55} \mathrm{~s}^{4} \mathrm{~m}^{-1}$ (dotted curve). (b) Ripple relative amplitudes associated with the FOPA of Fig. 2 (dashed curve) and the two FOPAs of Fig. 3(b) with positive $\beta_{4}$ (solid line) and negative $\beta_{4}$ (dotted curve).

In conclusion, we have investigated the nature of ripples in the flat-gain region of $2 \mathrm{P}$ FOPAs. These ripples may be large enough to lead to impairments in telecommunication systems. By looking at the eigenvalues, we were able to predict their pseudoperiods. To reduce the ripples amplitudes, we have shown that the 2P-FOPA should be designed such that the gain within the flat region between the two pumps is larger than the gain in the outer band. With positive $\beta_{4}$ fibers this design is straightforward. However, most conventional fibers have negative $\beta_{4}$. In this case, the gain between the two pumps should be maximized in order to reduce the ripples.

This work has been funded by the Conseil Regional de Franche-Comté, the European Regional Development Fund (ERDF), and the UK's Engineering and Physical Sciences Research Center (EPSRC). The authors gratefully thank J. M. Chavez Boggio and coworkers for sharing their experimental results.

\section{References}

1. M. E. Marhic, Fiber Optical Parametric Amplifiers, Oscillators and Related Devices (Cambridge U. Press, 2007).

2. C. J. McKinstrie, S. Radic, and A. H. Gnauck, Opt. Photon. News 18(3), 34 (2007).

3. M. C. Ho, M. E. Marhic, K. K. Y. Wong, and L. G. Kazovsky, J. Lightwave Technol. 20, 469 (2002).

4. A. Vedadi, A. Mussot, E. Lantz, H. Maillotte, and T. Sylvestre, Opt. Commun. 267, 244 (2006).

5. M. E. Marhic, K. K.-Y. Wong, and L. G. Kazovsky, J. Opt. Soc. Am. B 20, 2425 (2003).

6. M. E. Marhic, Y. Park, F. S. Yang, and L. G. Kazovsky, Opt. Lett. 21, 1354 (1996).

7. J. M. Chavez Boggio, J. D. Marconi, S. R. Bickham, and H. L. Fragnito, Opt. Express 15, 5288 (2007).

8. M. Yu, C. J. McKinstrie, and G. P. Agrawal, Phys. Rev. E 48, 2178 (1993).

9. L. Provino, H. Maillotte, E. Lantz, T. Sylvestre, and J. M. Dudley, in Conference on Lasers and Electro-Optics Europe, IEEE Conference Digest (IEEE, 2000), paper CtuL4.

10. C. J. McKinstrie and S. Radic, Opt. Lett. 27, 1138 (2002).

11. A. Vedadi, E. Lantz, H. Maillotte, and T. Sylvestre, in 2008 IEEE/LEOS Winter Topical Meeting Series (IEEE, 2008), paper TuB 1.2.

12. M. E. Marhic, A. A. Rieznik, and H. L. Fragnito, J. Opt. Soc. Am. B 25, 22 (2008). 